\title{
Fault Diagnosis System of Induction Motors Based on Multiscale Entropy and Support Vector Machine with Mutual Information Algorithm
}

\author{
Shuang Pan, ${ }^{1}$ Tian Han, ${ }^{1}$ Andy C. C. Tan, ${ }^{2}$ and Tian Ran Lin ${ }^{3}$ \\ ${ }^{1}$ School of Mechanical Engineering, University of Science and Technology Beijing, 30 Xueyuan Road, Haidian District, \\ Beijing 100083, China \\ ${ }^{2}$ Faculty of Engineering, Universiti Tunku Abdul Rahman, Sungai Long Campus, Kajang, Malaysia \\ ${ }^{3}$ School of Mechanical Engineering, Qingdao Technological University, 777 Jialingjian Road, Qingdao 266520, China
}

Correspondence should be addressed to Tian Ran Lin; trlin@qtech.edu.cn

Received 10 March 2015; Revised 26 August 2015; Accepted 26 August 2015

Academic Editor: Mohammad Elahinia

Copyright (C) 2016 Shuang Pan et al. This is an open access article distributed under the Creative Commons Attribution License, which permits unrestricted use, distribution, and reproduction in any medium, provided the original work is properly cited.

\begin{abstract}
An effective fault diagnosis method for induction motors is proposed in this paper to improve the reliability of motors using a combination of entropy feature extraction, mutual information, and support vector machine. Sample entropy and multiscale entropy are used to extract the desired entropy features from motor vibration signals. Sample entropy is used to estimate the complexity of the original time series while multiscale entropy is employed to measure the complexity of time series in different scales. The entropy features are directly extracted from the nonlinear, nonstationary induction motor vibration signals which are then sorted by using mutual information so that the elements in the feature vector are ranked according to their importance and relevant to the faults. The first five most important features are selected from the feature vectors and classified using support vector machine. The proposed method is then employed to analyze the vibration data acquired from a motor fault simulator test rig. The classification results confirm that the proposed method can effectively diagnose various motor faults with reasonable good accuracy. It is also shown that the proposed method can provide an effective and accurate fault diagnosis for various induction motor faults using only vibration data.
\end{abstract}

\section{Introduction}

Induction motors are one of the most critical machine components in industry today. A breakdown of the motor can lead to the shutdown of a whole production line and results in expensive downtime. It is thus important to have a reliable condition monitoring $(\mathrm{CM})$ and fault diagnosis system in place to continuously monitor the healthy condition of such machine components. Early fault detection of the motor can increase the machine performance and availability, reduce the consequential damage, prolong the machine useful lifespan, reduce spare parts inventories, prevent unexpected breakdown, and ensure timely maintenance schedules [1]. Induction motor fault diagnosis has attracted considerable attention from researchers in recent years.

Table 1 summarizes the most typical faults and their occurrence possibilities of induction motors from the statistical studies conducted by the Institute of Electrical and
Electronics Engineers (IEEE) and by Electric Power Research Institute (EPRI) through General Electric Corporation [2]. The study identified that faulty bearings are the most common induction motor fault (representing more than $40 \%$ of motor faults). This is followed by the stator fault (over 28\%), rotor fault (over 8\%), and other unspecified faults (more than $12 \%)$.

Several CM techniques such as vibration analysis, acoustic analysis [3], motor current signature analysis [4-7], electromagnetic field monitoring [8], chemical analysis, and temperature analysis $[9,10]$ have been developed in the last two decades for the detection and diagnosis of the induction motor faults. Vibration technique is one of the most frequently employed techniques for motor faults detection due to its ease of use, high accuracy, and reliability. Nevertheless, vibration signals from induction motors are usually nonlinear and nonstationary and have weak signal energies. Traditional 
TABLE 1: Typical induction motor faults and the corresponding occurrence possibilities.

\begin{tabular}{lcccc}
\hline & $\begin{array}{c}\text { Bearing } \\
\text { faults }\end{array}$ & $\begin{array}{c}\text { Stator } \\
\text { faults }\end{array}$ & $\begin{array}{c}\text { Rotor } \\
\text { faults }\end{array}$ & Others \\
\hline IEEE & $42 \%$ & $28 \%$ & $8 \%$ & $22 \%$ \\
EPRI & $40 \%$ & $38 \%$ & $10 \%$ & $12 \%$ \\
\hline
\end{tabular}

frequency domain analysis techniques such as fast Fourier transform (FFT) are thus not suitable to be directly employed for the analysis of motor vibration signals. To overcome this problem, other data analysis techniques such as short time Fourier transform, wavelet transform, and Hilbert-Huang transform (HHT) techniques as well as artificial intelligent techniques such as neural network have been developed. For instance, Talhaoui et al. [11] used a combined FFT and discrete wavelet transform (DWT) technique to evaluate faults due to broken rotor bars of an induction motor. Roveri et al. $[12,13]$ proposed a HHT-based method for damage detection of a bridge structure under moving loads. EMD technique, the main functionality of HHT, has attracted great attention in recent years and has been widely employed for fault diagnosis of rotating machinery. EMD technique still has several shortcomings though such as mode mixing and the end effect. To alleviate the mode mixing problem, an improved method, ensemble empirical mode decomposition (EEMD), was developed [14], which has been successfully employed by Lei et al. [15] for fault diagnosis of rotating machinery. On the other hand, Liang et al. [16] used a combination of power spectrum, cepstrum, bispectrum, and neural network for the data analysis and fault diagnosis of induction motors. Seera et al. [17] employed a hybrid computer model comprising a Fuzzy Min-Max (FMM) neutral network and a Classification and Regression Tree (CART).

Entropy, a measure of the uncertainty of a process [18] which was first applied in thermodynamics, has been successfully employed to analyze the physiological, biological, and electrocardiogram signals [19]. Lin et al. [20] utilized a multiscale entropy method combining with wavelet denoising to detect a motor shaft misalignment. Zheng et al. [21] developed a rolling bearing fault diagnosis method based on local characteristic-scale decomposition (LCD) and fuzzy entropy (FuzzyEn). Zhu et al. [22] proposed a feature extraction method based on hierarchical entropy (HE) to effectively identify various faulty bearing conditions. Sample entropy (SampEn) and multiscale entropy (MSE) were introduced in their paper to extract features from the vibration data.

The variation of parameters used in the entropy algorithms can lead to redundant entropy features causing excessive training time and affect the system accuracy. To overcome this problem, a mutual information (MI) technique originally proposed by [23] is adopted in this study to select the most effective features from the extracted entropies to improve the efficiency and accuracy of the induction motor fault diagnosis process.

Once a fault feature vector has been selected, a multifault classifier is needed to identify the faulty conditions of an induction motor. Several pattern recognition techniques

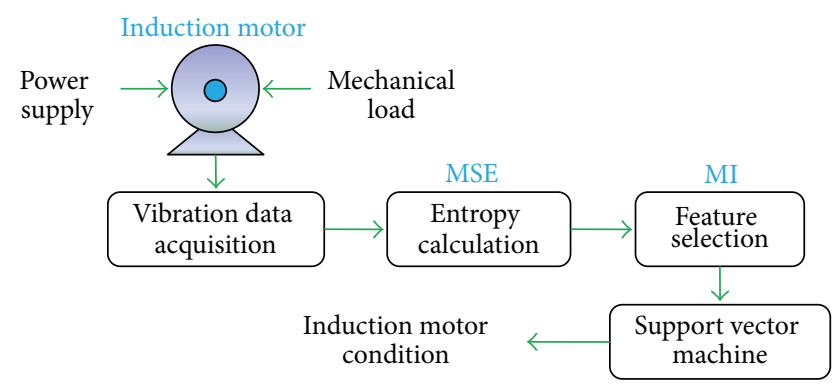

FIgURE 1: The flowchart of the fault diagnosis algorithm for induction motors.

such as artificial neural networks (ANN) and support vector machines (SVM) have been developed in recent years for mechanical fault classification. SVM has recently been successfully applied to face detection, object detection and recognition, pattern recognition, and information and image retrieval due to its high accuracy and good generalization with a smaller number of samples [24]. It is used in this study for fault diagnosis of an induction motor due to the consideration that large fault samples are difficult to obtain in practice. An induction motor fault test rig is also built in the laboratory to validate the fault diagnosis technique proposed in this paper.

The remainder of the paper is organized as follows. Section 2 briefly discusses the concept of SampEn, MSE, MI, and SVM algorithms used in this paper and introduces the fault diagnosis system proposed in the study. Section 3 describes the experimental setup of the induction motor fault simulation test rig. Section 4 presents the analysis of the experimental data for motor fault diagnosis using the proposed method. The main findings from this study are summarized in Section 5.

\section{The Procedure of the Proposed Motor Fault Diagnosis Method}

The flowchart of the proposed induction motor diagnosis algorithm is shown in Figure 1. The first step of the algorithm is to calculate the entropy values from the vibration data acquired from an induction motor utilizing SampEn and MSE. Mutual information technique is then employed to select the effective entropy features in the second step. SVM is applied to discriminate the motor faults in the last step from the selected entropy features.

For a better understanding of the theoretical foundation and process, the procedure and techniques involved in each step of the proposed fault diagnosis algorithm are briefly discussed and explained in the following text.

2.1. Sample Entropy. Online diagnosis systems are gaining popularity in industry applications nowadays due to their capability to detect incipient faults at an early stage. However, directly measured CM signals are not suitable for online analysis as a small dataset is not good enough for a reliable diagnosis. On the other hand, a larger dataset poses a burden 
for data processing. Feature extraction is thus a critical initial step in a monitoring and fault diagnosis system. The accuracy of feature extraction has a direct effect on the accuracy of the final diagnosis outcome. An important criterion is that a feature extraction method should be able to preserve the critical information for decision-making. Based on this consideration, SampEn and MSE algorithms are adopted in this paper to extract the usable effective features from the vibration data.

Pincus [25] proposed a statistical measure, Approximate Entropy (ApEn), to quantify the regularity in a short and noisy time series. ApEn reflects the likelihood of sequences that are close to each other. That is to say, within given tolerance $r, m$ consecutive data points remain close when one more data point is known [26]. In general, lower values of ApEn reflect more regular time series, while higher values are associated with less predictable time series. ApEn measures the time series for similar segments, and a large number of such segments yield a small value of ApEn. Importantly, the ApEn algorithm counts each sequence as matching itself to avoid the occurrence of $\ln (0)$ in the calculations. This condition has led to bias in the calculation of ApEn. In practice, the bias causes ApEn to lack two important expected properties: (1) ApEn is heavily dependent on the record length and is uniformly lower than the expected value for short records and (2) it lacks relative consistency; that is to say, if ApEn of one dataset is higher than that of another, it should, but does not, remain higher for all tested conditions.

To overcome the shortcomings of ApEn, Richman and Moorman [27] developed and characterized a new family of statistical measures, sample entropy (SampEn), which do not count the self-matches. SampEn is precisely the negative natural logarithm of the conditional probability that two sequences with similar $m$ points remain similar at the next point, where self-matches are not included in calculating the probability. Thus, a lower value of SampEn also indicates more self-similarity in a time series. In addition to the benefit of eliminating self-matches, SampEn algorithm is also simpler and uses only about one-half of the time compared with ApEn algorithm for the same calculation. SampEn is largely independent of the data record length and displays relative consistency under various circumstances. Mathematically, SampEn algorithm can be summarized as follows.

Let $X$ be a time series of length $N: X=$ $\{x(1), x(2), \ldots, x(N)\}$ :

(1) Construct template vectors with dimension $m$ by using the following equation:

$$
\begin{array}{r}
X_{i}^{m}=[x(i), x(i+1), \ldots, x(i+m-1)], \\
\quad i=1,2, \ldots, N-m .
\end{array}
$$

(2) A match occurs when the distance between two template vectors $\left(X_{i}^{m}, X_{j}^{m}\right)$ is smaller than predefined tolerance $r$. A schematic illustration of the distance

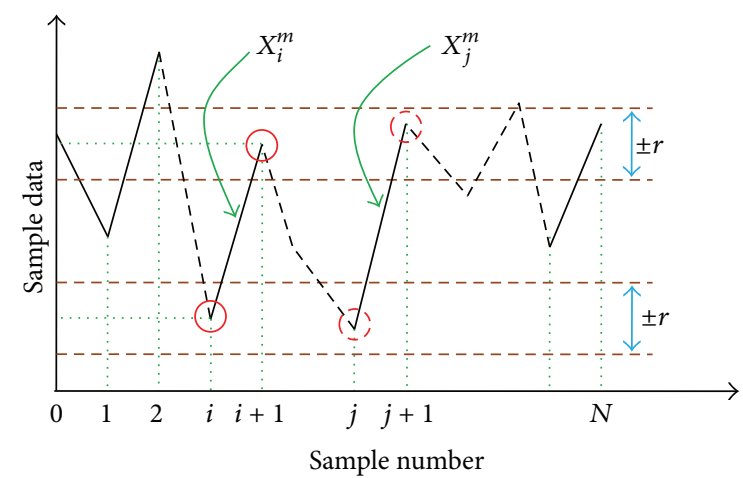

FIGURE 2: A schematic illustration of the distance calculation $(m=$ 2).

calculation is shown in Figure 2. The distance between the two vectors is calculated using

$$
\begin{aligned}
d\left[X_{i}^{m}, X_{j}^{m}\right]=\max _{k=0,1, \ldots, m-1}[|x(i+k)-x(j+k)|], & \\
& i, j=1,2, \ldots, N-m, i \neq j .
\end{aligned}
$$

(3) Let $n_{i}^{m}(r)$ be the number of distances within $r$ and let $B_{i}^{m}(r)$ be the total number of $m$-dimension matched vector pairs:

$$
B_{i}^{m}(r)=\frac{n_{i}^{m}(r)}{(N-m-1)}, \quad i=1,2, \ldots, N-m .
$$

(4) Define the average value of $B_{i}^{m}(r)$ such that

$$
B^{m}(r)=\sum_{i=1}^{N-m} \frac{B_{i}^{m}(r)}{(N-m)} .
$$

(5) Repeat steps (1)-(4) for $m+1$, and $B^{m+1}(r)$ can be obtained.

(6) For finite $N$, SampEn is defined as the logarithm of the ratio of $B^{m+1}(r)$ and $B^{m}(r)$ :

$$
\begin{aligned}
\operatorname{SampEn}(m, r, N) & =-\ln \left[\frac{B^{m+1}(r)}{B^{m}(r)}\right] \\
& =\ln B^{m}(r)-\ln B^{m+1}(r),
\end{aligned}
$$

where $N$ is the length of the time series, $m$ is the dimension of sequences to be compared, and $r$ is the tolerance level for accepting matches. It is convenient to set the tolerance as $r \times \mathrm{SD}$, where $\mathrm{SD}$ is the standard deviation of the dataset. It is worth noting that the parameters $m, r$, and $N$ must be fixed for each calculation.

2.2. Multiscale Entropy (MSE). Regularity in a time series is sometimes presented as coarse granularity; thus, the time series needs to be explored using different scales. Costa et al. 

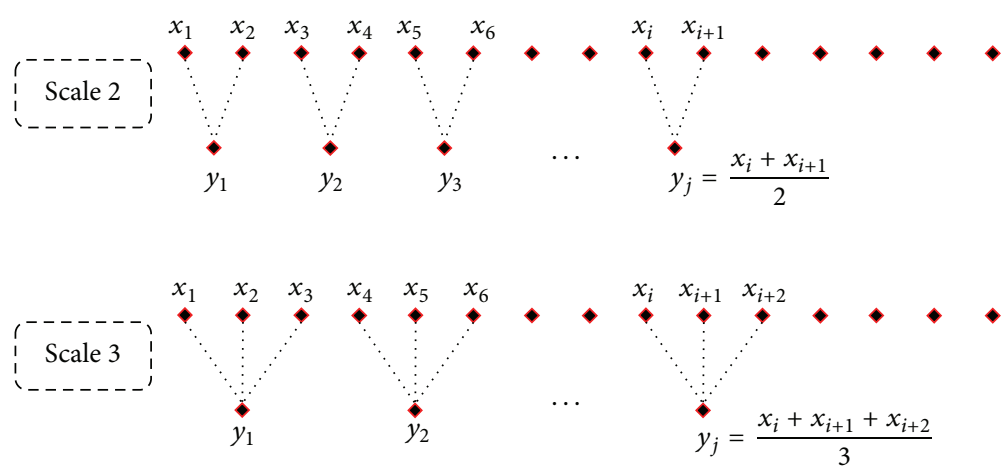

FIGURE 3: A schematic illustration of the coarse-graining procedure.

[28] suggested that it is generally difficult to distinguish the interbeat interval time series of different diseased and healthy states when only a single-scale SampEn is used. Therefore, the concept of MSE is proposed to resolve this difficulty. For the sake of brevity, only the two main steps of MSE algorithm are discussed here:

(1) A coarse-graining step, which is used to obtain the representations of the original time series on different time scales.

(2) The SampEn step, which is used to quantify the regularities of the coarse-grained time series.

To obtain the coarse-grained time series at a scale factor, $\tau$, the original time series is divided into nonoverlapping windows of length $\tau$, and the data points inside each window are averaged. A coarse-grained time series in MSE is defined using

$$
y_{j}(\tau)=\frac{1}{\tau} \sum_{i=(j-1) \tau+1}^{j \tau} x(i), \quad 1 \leq j \leq \frac{N}{\tau} .
$$

For $\tau=1$, the coarse-grained time series is the original time series. As $\tau$ increases, the length of the resulting coarsegrained time series decreases. Figure 3 describes $\tau$ coarsegrained time series divided from the original time series for a scale factor of $\tau$. In the conventional MSE algorithm, the MSE at a scale factor of $\tau$ is defined as the SampEn of the first coarse-grained time series. The SampEn for each coarsegrained time series is calculated and plotted as a function of the scale factor $\tau$ in the final step to complete the procedure of multiscale entropy analysis.

2.3. Mutual Information for Feature Selection. The extracted SampEn and MSE features are able to discriminate different types of motor faults. Nevertheless, a feature vector with high dimension will be very time consuming to analysis. Therefore, it is our aim to reduce the feature space by selecting only the most effective features containing the fault information to improve the performance of SVM classification and the effectiveness of fault diagnosis.
Mutual information (MI) is a measure of the variables' mutual dependence. One major advantage of MI for feature selection is its ability to detect nonlinear relationships between variables. MI can also be defined for groups of variables (or equivalently for multidimensional variables), which allows one to take the joint relevance and redundancy of features into account during the feature selection process. Mutual information $I(X, Y)$ is the amount of uncertainty in $X$ due to the knowledge of $Y$. Mathematically, MI can be calculated as [23]

$$
I(X, Y)=\sum_{x, y} p(x, y) \log \frac{p(x, y)}{p(x) p(y)},
$$

where $p(x, y)$ is the joint probability distribution function of $X$ and $Y$ and $p(x)$ and $p(y)$ are the marginal probability distribution functions for $X$ and $Y$. MI is employed in this study to select a subset of relevant features, which considers feature-class mutual information to determine an optimal set of features by sorting entropy features in descending order. It is noted that MI has not been employed previously for induction motor fault diagnosis. It is used in this study to select the effective entropy features closely related to a motor fault.

2.4. Support Vector Machine. SVM algorithm is capable of handling large feature spaces since the training of SVM is carried out in such a manner that the dimension of classified vectors does not have any distinct influence on the performance of SVM as it does on the performance of other conventional classifiers. This property is very useful for fault classification since the number of features for fault diagnosis need not be limited. Furthermore, SVM-based classifier also has better generalization properties than other conventional classifiers attributed to the minimum structural misclassification risk when training the SVM classifier, whereas other traditional classifiers are usually trained to minimize the empirical risk $[29,30]$. SVM can also be used in nonlinear classification tasks with the selection of the kernel functions. The definition of a legitimate kernel function is given by Mercer's theorem which states that the function must be continuous and positive definite. Any function that satisfies 
TABLE 2: Formulation of kernel functions.

\begin{tabular}{ll}
\hline Kernel & $K\left(\mathbf{x}_{i}, \mathbf{x}_{j}\right)$ \\
\hline Linear function & $K\left(\mathbf{x}_{i}, \mathbf{x}_{j}\right)=\mathbf{x}_{i}^{T} \times \mathbf{x}_{j}$ \\
\hline Polynomial function & $K\left(\mathbf{x}_{i}, \mathbf{x}_{j}\right)=\left(g \mathbf{x}_{i}^{T} \times \mathbf{x}_{j}+r\right)^{d}, \quad g>0$ \\
\hline Radial basis function & $K\left(\mathbf{x}_{i}, \mathbf{x}_{j}\right)=\exp \left(\frac{-\left\|\mathbf{x}_{i}-\mathbf{x}_{j}\right\|}{2 g^{2}}\right)$ \\
\hline
\end{tabular}

Mercer's theorem can be used as a kernel function to compute the dot product in the feature space. Various kernel functions such as linear, polynomial, and radial basis functions are used in SVM classification. The selection of an appropriate kernel function is very important in the classification process as the kernel defines the feature space in which the training set examples are classified. Linear, polynomial, and radial basis functions are evaluated and formulated in this paper and listed in Table 2, where parameter $g$ is the kernel parameter.

The fault diagnosis of an induction motor is a multifault classification since there are various fault types for the motor (also see Table 1). Commonly used techniques for multiclass classification include "one-against-all" or "one-against-one" techniques. The "one-against-one" technique is adopted in this paper. For this technique, if $k$ is the number of classes, it constructs $k(k-1) / 2$ classifiers and each one is trained on the data from two classes. For the training data from the $i$ th and $j$ th classes, the two-class classification problem is solved as follows:

$$
\begin{array}{cl}
\text { Minimize: } & \frac{1}{2}\left\|\omega^{i j}\right\|^{2}+c \sum_{t} \xi_{t}^{i j}\left(\omega^{i j}\right)^{T}, \\
\text { subject to: } & \left(\omega^{i j}\right)^{T} \varphi\left(x_{t}\right)+b^{i j} \geq 1-\xi_{t}^{i j}, \\
& \left(\omega^{i j}\right)^{T} \varphi\left(x_{t}\right)+b^{i j} \geq \xi_{t}^{i j}-1, \\
& \xi_{t}^{i j} \geq 0,
\end{array}
$$

where parameter $c$ is the penalty parameter of the error classification. The Max Win Strategy [22] is utilized during the classification as follows: if $\operatorname{sign}\left(\left(\omega^{i j}\right)^{T} f\left(x_{t}\right)+b^{i j}\right)$ indicates that $x$ is in the $i$ th class, the vote for the $i$ th class is increased by one; otherwise, the $j$ th class is increased by one. Then, $x$ is designated to be in a class with the maximum number of votes.

The fault diagnosis procedures described in this section will be employed to analyze the experimental data from an induction motor fault simulation test rig to be elaborated in the following sections.

\section{A Description of the Fault Simulation Test Rig and the Experimental Setup}

The induction motor test rig was designed to carry out the fault simulation as shown in Figure 4. It consists of an induction motor, a pulley and pulley belt, a shaft and a fan

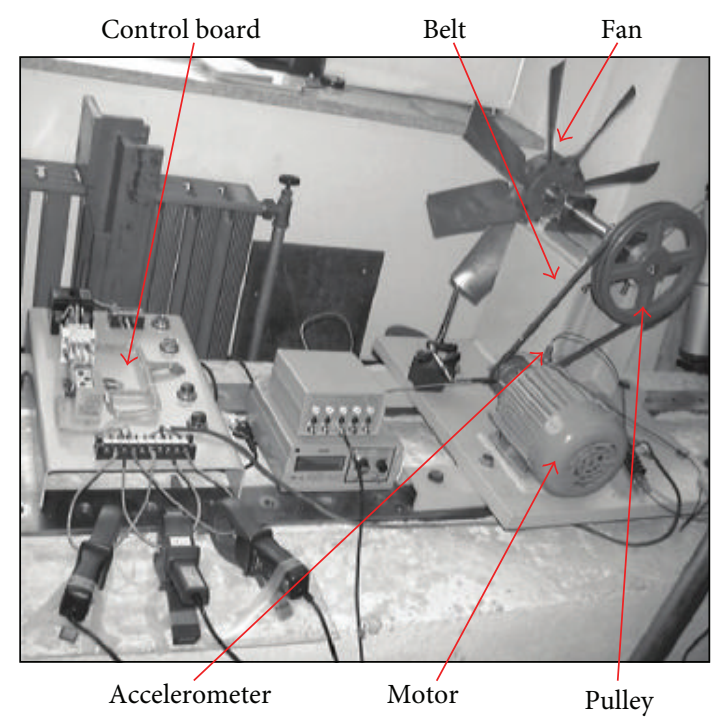

FIGURE 4: A description of the fault simulation test rig and the instrumentation.

with changeable pitch blades, bearings, and the supporting structure.

Six $0.5 \mathrm{~kW}, 60 \mathrm{~Hz}, 4$-pole induction motors (1 healthy and 5 faulty) were used in the experiment to generate the required dataset under the full-load condition. Data from the healthy motor is used as a benchmark for comparison with the experimental data from other faulty motors. The faulty motors each have a specific fault: (1) unbalance rotor (MUN), (2) broken rotor bar (BRB), (3) faulty bearing (outer race, FBO), (4) bowed rotor (BR), and (5) eccentricity (angular misalignments (AMIS) and parallel misalignments (PMIS)) as shown in Figure 5. Furthermore, phase unbalance (PUN) is also simulated in the experiment by adding windings on one of the phases of the healthy motor (NOR). The faulty conditions of these motors are also listed in Table 3.

Three accelerometers are used in the experiment to measure the vibration signals in the three coordinate directions (i.e., horizontal, vertical, and axial) of the motors to evaluate the effectiveness and the functionality of the proposed fault diagnosis algorithm. The frequency range of the vibration signals in the setup is up to $5 \mathrm{kHz}$ and the total number of data samples acquired is 16384. Figure 6 displays a set of time waveforms of the measured vibration signals for the simulated induction motor faults investigated in this study. It is observed that the difference between the vibration time waveforms for the normal, unbalance rotor, broken rotor bar, and phase unbalance cases is insignificant. For these cases, the waveforms appear to be slightly distorted from a pure sine wave having the fundamental frequency of the shaft rotating speed. For the eccentricity cases, the waveforms are mainly composed of the vibration from the fundamental shaft rotating frequency and are superimposed with vibration from higher order components. For the bowed rotor and faulty bearing cases, the vibration waveforms are largely distorted by impact components. 


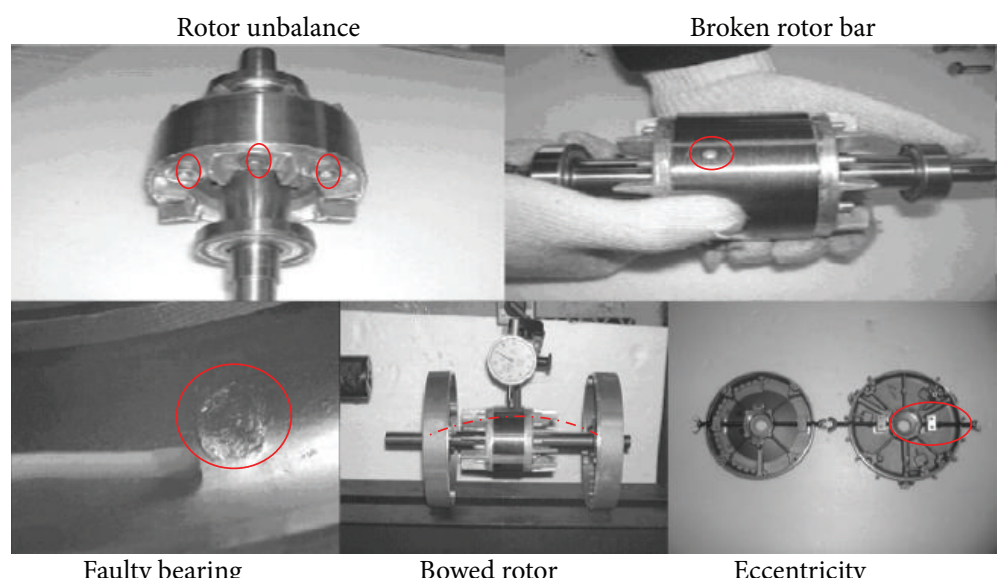

FIgURE 5: Typical induction motor faults.

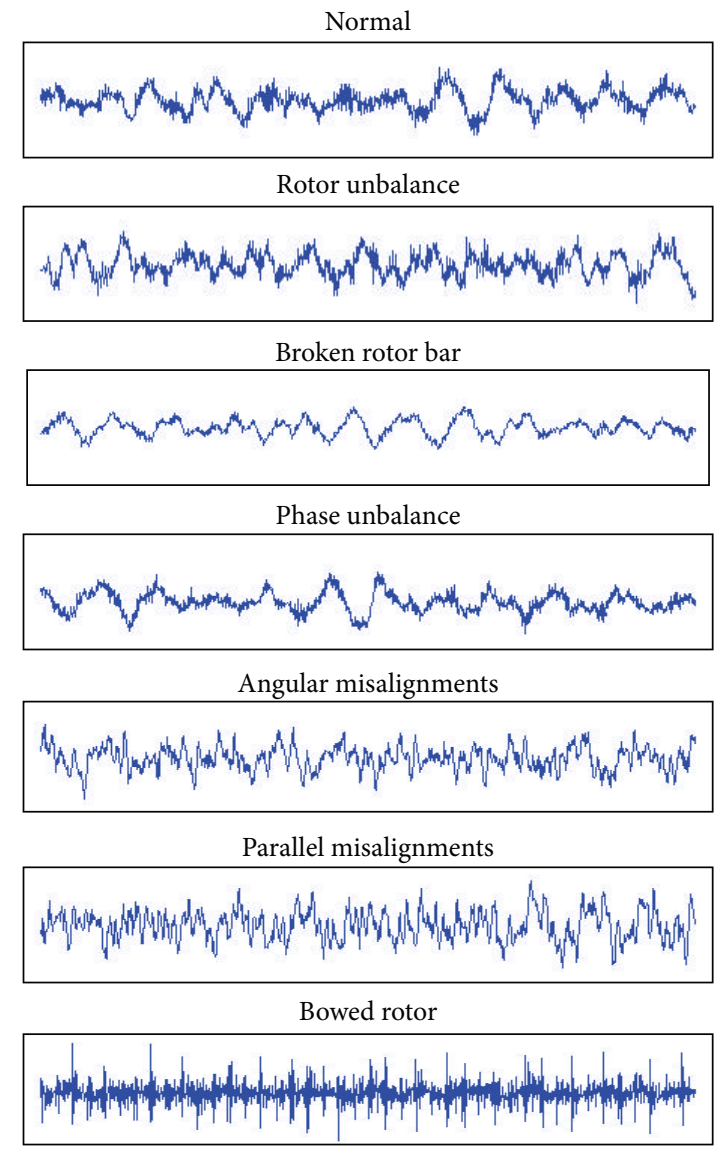

Faulty bearing

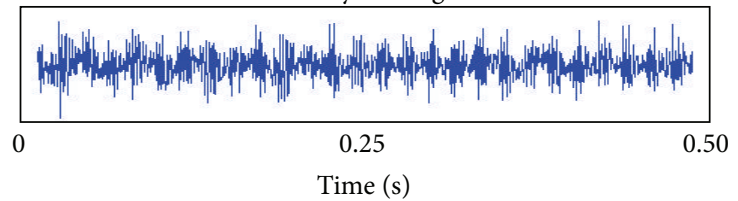

FIGURE 6: The representative vibration waveforms of the induction motor faults. 
TABLE 3: A description of the faulty induction motors.

\begin{tabular}{lcc}
\hline Fault condition & Fault description & Others \\
\hline Unbalance rotor (MUN) & Unbalance mass on the rotor: $8.4 \mathrm{~g}$ & Total number of 34 bars \\
Broken rotor bar (BRB) & Number of broken bars: 12 & NSK-6203 \\
Faulty bearing (FBO) & Spall on the outer race & Air gap: 0.25 mm \\
Bowed rotor (BR) & Rotor deflection: $0.075 \mathrm{~mm}$ & Adjusting the bearing pedestal \\
Eccentricity (AMIS) & Angular misalignments & Adjusting the bearing pedestal \\
Eccentricity (PMIS) & Parallel misalignments & Using the normal motor \\
Phase unbalance (PUN) & Adding windings on one of the phases
\end{tabular}

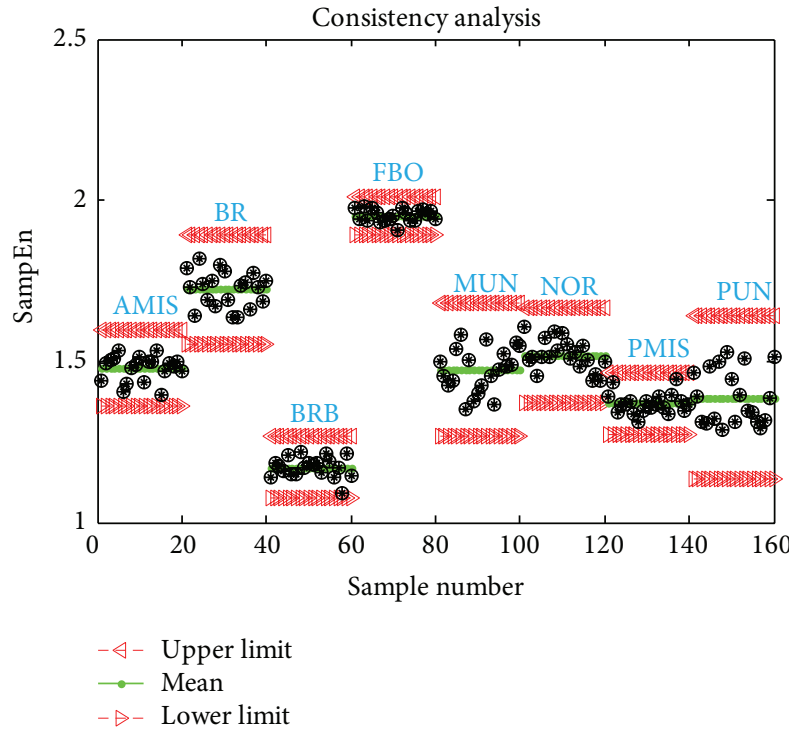

(a)

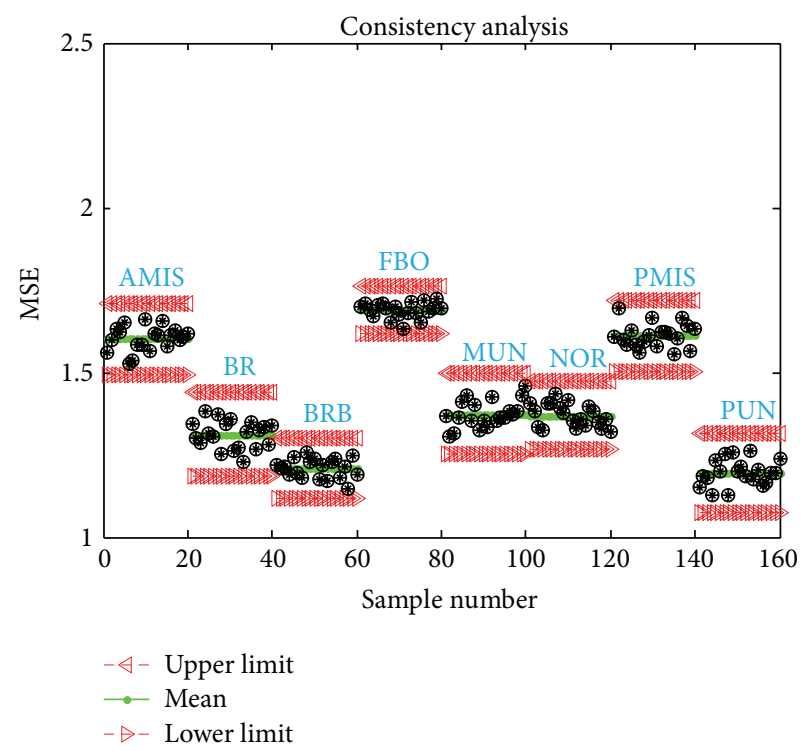

(b)

FIGURE 7: Consistency analysis of the two chosen entropy features. (a) Consistency analysis of SampEn (tolerance, $r=0.2)$ and (b) consistency analysis of MSE (scale factor, $\tau=2$ ).

\section{Application of the Proposed Algorithm for Motor Fault Diagnosis}

4.1. Consistency Analysis for Each Fault Condition. An effective feature should be able to distinguish different induction motor fault conditions and satisfy the consistency requirement for each induction motor fault. A consistency analysis is thus required before the use of entropy features for induction motor fault diagnosis since motor vibration data typically contains nonlinear components and noise. Random errors in the experiment can cause variation of the calculated entropy feature values. A large variation of the calculated entropy values will render the features inappropriate for fault classification. The " $3 \mathrm{~s}$ " rule in statistics is adopted in this study to evaluate the consistency of the two entropy features, SampEn and MSE.

Figure 7 shows the consistency analysis of the two entropy features for the simulated motor faults. The black dots are the entropy values of 20 data samples for each simulated motor fault, and the green line represents the mean entropy value for each fault. The analysis of the distribution of the two entropy features using the " $3 \mathrm{~s}$ " criteria indicates that both SampEn and MSE satisfy the consistency requirement.

4.2. Entropy Calculation and Feature Selection. As described in Section 2, three parameters, namely, the tolerance $r$, the dimension of sequences $m$, and the length of the time series $N$, are used in the calculation of SampEn. The length of each data sample is automatically determined by the data acquisition setup, which is $N=4096$. The dimension of sequences, $m=2$, is used in this calculation. The tolerance $r$ is commonly expressed as a fraction of the standard deviation (SD) of the time series that affects the SampEn value. A small $r$ will lead to a poor conditional probability, while a large $r$ will result in losing too much system information. In principle, $r$ should be a value that minimizes the effect of noise on the calculation of the SampEn. The value of $r$ chosen in this study ranges between $0.1 \mathrm{SD}$ and $0.7 \mathrm{SD}$. Figure 8 illustrates 


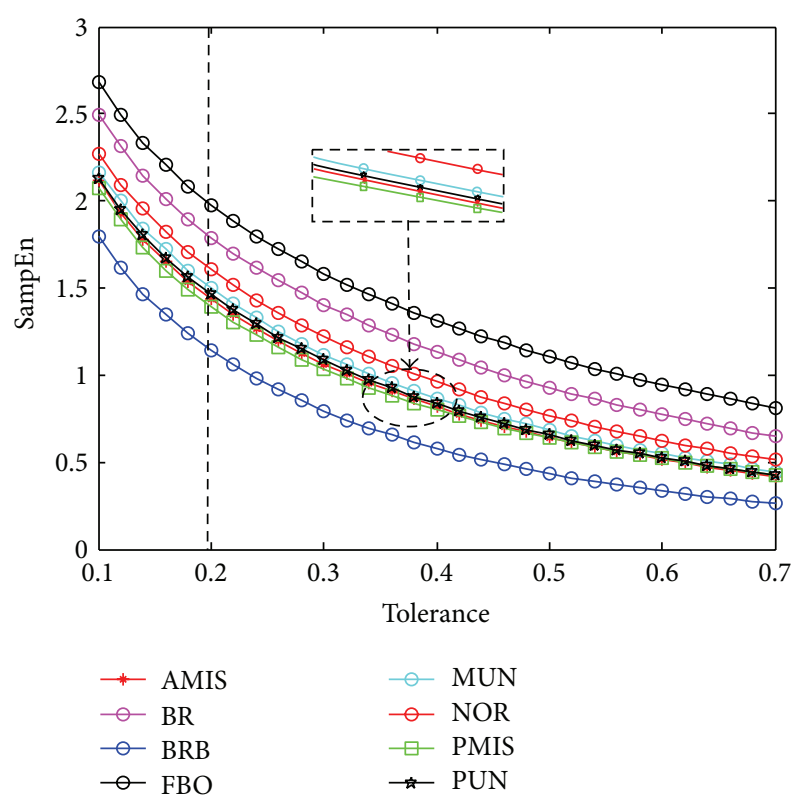

FIgURE 8: The effect of chosen tolerance values on the entropy feature, SampEn.

the effect of $r$ value on the calculated SampEn value for the motor faults simulated in this study. It is shown that the value of SampEn decreases steadily for increasing $r$ value. The calculated SampEn for the faulty bearing (FBO) case serves as the upper bound for all cases attributed to the impact impulses and high frequency components in the vibration signal (see Figure 6). On the contrary, the calculated SampEn for the broken rotor bar (BRB) case serves as the lower bound of all cases as a result of the least noisy low frequency vibration waveform recorded for this case (Figure 6). It is further observed that the calculated SampEn for the bowed rotor (BR), BRB, FBO, and normal (NOR) cases can be clearly distinguished from each other and thus can be used directly for motor fault classification. On the other hand, the entropy values for the eccentricity (AMIS, PMIS), unbalance rotor (MUN), and phase unbalance (PUN) cases are clustered together which are difficult to distinguish from each other. Further efforts are thus required to derive a more suitable (separable) feature for these later cases for fault classification.

MSE features are therefore selected to serve this purpose to overcome the limitation of the SampEn features. The parameter values used in MSE feature extraction are $m=2$, $r=0.2 \mathrm{SD}$, and $N=4096$ with a maximum scale factor of $\tau=10$. Figure 9 compares the time waveforms of the coarse-grained time series for AMIS and FBO cases using the first five scale factors: $\tau=1,2, \ldots 5$. It is shown that the AMIS waveform becomes more complex with increasing scale factor while the FBO waveform is less complex for larger scale factors.

Figure 10 compares the MSE values for the simulated motor faults investigated in this study for the scale factor ranging from 1 to 10 . For the scale factor of 1 , the calculated MSE value is the same as the SampEn value. The MSE value for the FBO case is the highest and the MSE value for the BRB
TABLE 4: Entropy features selection using MI algorithm.

\begin{tabular}{lcccc}
\hline Features number & Entropy & Dimension & Tolerance & Scale factor \\
\hline 1 & MSE & $m=2$ & $r=0.2$ & $\tau=1$ \\
2 & MSE & $m=2$ & $r=0.2$ & $\tau=2$ \\
3 & MSE & $m=2$ & $r=0.2$ & $\tau=3$ \\
4 & MSE & $m=2$ & $r=0.2$ & $\tau=5$ \\
5 & MSE & $m=2$ & $r=0.2$ & $\tau=7$ \\
\hline
\end{tabular}

TABLE 5: Classification accuracy using different SVM kernel functions.

\begin{tabular}{lccc}
\hline Number & Kernel function & $\begin{array}{c}\text { Parameters of } \\
\text { svmtrain }\end{array}$ & $\begin{array}{c}\text { Classification } \\
\text { accuracy }\end{array}$ \\
\hline 1 & $\begin{array}{c}\text { Linear function } \\
\text { Polynomial } \\
\text { function } \\
\text { Radial basis } \\
\text { function }\end{array}$ & $-c 8-g 4-\tau 0$ & $91.25 \%(73 / 80)$ \\
3 & $-c 8-g 4-\tau 1$ & $91.25 \%(73 / 80)$ \\
\hline
\end{tabular}

case is the lowest under this condition. It is further shown that the MSE values for the BR and FBO cases decrease rapidly as the scale factor increases due to the reduced complexity of the waveforms (see Figure 9). Conversely, the MSE values for the AMIS and PMIS cases increase as the scale factor increases attributed to the increased complexity of the waveforms (see Figure 9). Starting from the scale factor, $\tau=4$, onward, the MSE values of the coarse-grained time series for AMIS and PMIS cases are much larger than those of PUN and MUN cases for the same scale factor. The features for these faulty cases can be clearly distinguishable for higher scale factors. This is particularly so for the three former cases. Therefore, these MSE features will be adopted to distinguish the fault conditions among AMIS, PMIS, and PUN cases in the fault classification.

In the next step, MI algorithm is applied to sort the order of the features according to their importance and relationship with the faults. The first five features are selected and listed in Table 4 .

4.3. Fault Classification. Three different kernel functions including linear function, polynomial function, and radial basis function of SVM are used in the fault classification in this study. A supervised mode is used for data training using the LIBSVM Matlab Toolbox. Figure 11 illustrates the classification accuracy using different kernel functions and as a function of the selected parameter value $g$ in the training. The classification efficiency for the three SVM models using the parameter values, $c=8$ (a cost parameter) and $g=4$ (a width parameter), is given in Table 5 for comparison. It is shown that the SVM model using the radial basis function predicts the measured values with the highest accuracy.

After the data training, the second phase of SVM algorithm is to test and validate whether the classifier has learnt from unseen examples in the training phase. A second-fold cross-validation method is applied to the 160 experimental 

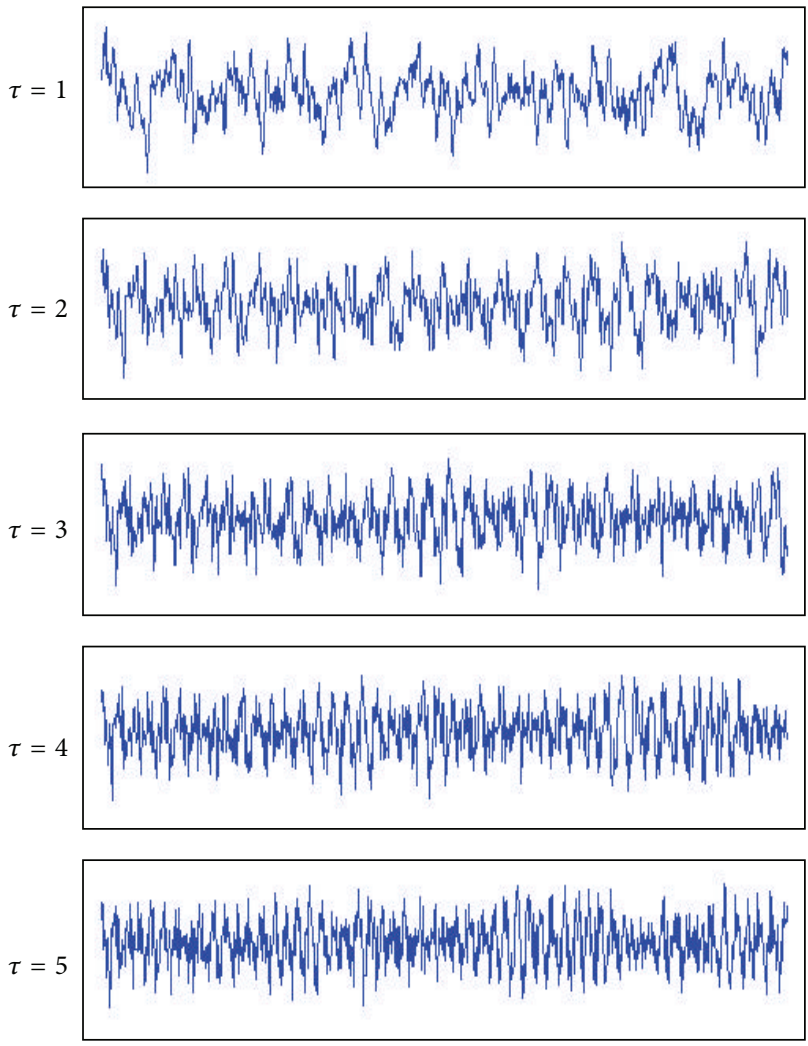

AMIS
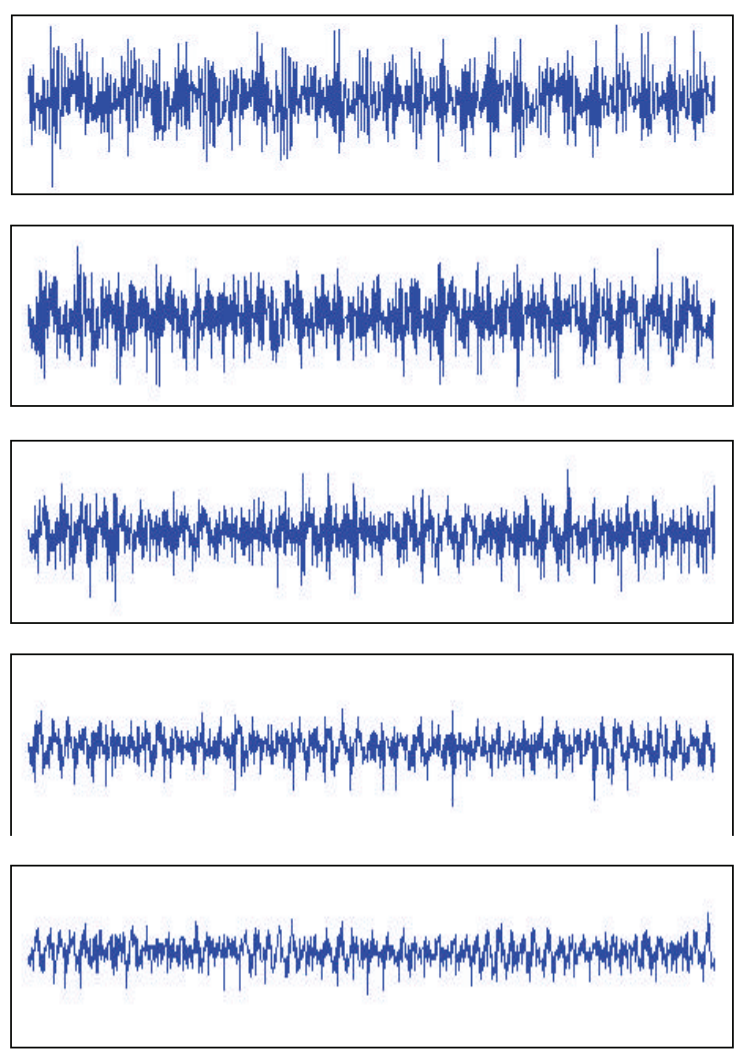

FBO

FIgURE 9: Time waveforms of coarse-grained time series.

TABLE 6: The second-fold test accuracy of the proposed method.

\begin{tabular}{lcccc}
\hline \multicolumn{2}{c}{ Dataset (160) } & Correctly classified & Wrongly classified & Classification accuracy \\
Training set & Test set & 74 & 6 & $92.50 \%$ \\
\hline Set-1 (80) & Set-2 (80) & 76 & 4 & $95.00 \%$ \\
Set-2 (80) & Set-1 (80) & & $\mathbf{9 3 . 7 5 \%}$ \\
& Average classification accuracy (all ten features) & 3 & $96.25 \%$ \\
Set-1 (80) & Set-2 (80) & 77 & 3 & $96.25 \%$ \\
Set-2 (80) & Set-1 (80) & 77 & $\mathbf{9 6 . 2 5} \%$ \\
& Average classification accuracy (the first five features using MI) & & \\
\hline
\end{tabular}

datasets for the validation of the proposed induction motor fault diagnosis system. In a $k$-fold cross-validation method, the dataset is divided into $k$ subsets, and the holdout method [31] is repeated $k$ times. At each time, $k-1$ subsets are used for training and $k$ th subset is used for testing. The average error for all $k$ trials is computed. The second-fold test accuracy of the proposed method is shown in Table 6. The average classification accuracy using the first five features and the MI algorithm is $96.25 \%$.

The prediction accuracy of the SVM model using the radial basis function is graphically illustrated in Figure 12. It is shown that the SVM classifier can identify the specific motor fault with $100 \%$ accuracy for most faults using the proposed method. Only three wrongly classified cases are found from the 160 datasets for the MUN and NOR cases due to the similarity of the waveform (see Figure 6) and entropy values (see Figure 10) between these two cases.

Figure 13 compares the classification accuracy of the proposed method and another method previously developed by the same authors [1]. It is shown that the method proposed in this study is as accurate or better for motor fault diagnosis in most cases except for one (NOR). The proposed method can identify 8 different operational conditions of induction motors with high accuracy which is an advantage over a previous work [20] where a single fault such as shaft misalignment was studied. The proposed method also differs 


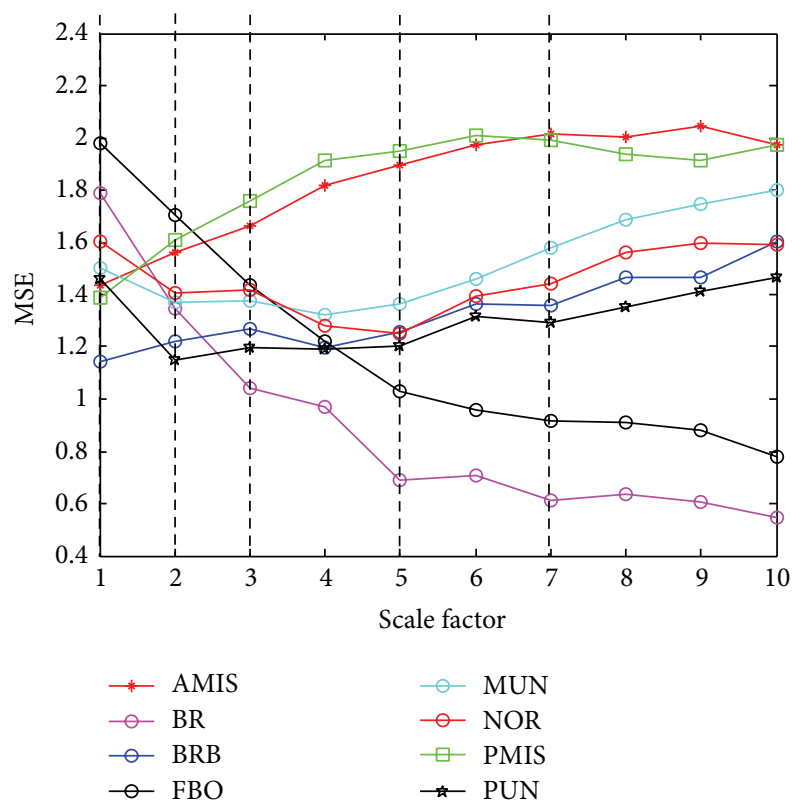

FIGURE 10: MSE of vibration signals.

from other previous works for multifault classification [3234] by using only vibration data and the two extracted entropy features, SampEn and MSE.

\section{Conclusion}

A fault diagnosis algorithm using two entropy features (SampEn and MSE), MI algorithm, and SVM classifier was proposed in this paper for induction motor fault diagnosis. The major steps and their sequential use in this algorithm are (1) extracting the entropy features from the nonlinear, nonstationary induction motor vibration signals; (2) sorting the features by using MI algorithm so that the elements in the feature vector are ranked according to their importance and relevant to the faults; (3) selecting the five most important features from the feature vectors and then classifying them using SVM method. The proposed classification and fault diagnosis technique was validated using the induction motor vibration data in the study. The classification results confirmed that the proposed fault diagnosis algorithm can effectively detect 8 various faults of the induction motor with an average accuracy of $96.25 \%$. The algorithm proposed in this study thus provides an effective tool for a reliable fault diagnosis of a multifault mechanical system such as an induction motor using only vibration CM data. The study presented in this paper highlights the notions that (1) entropy features are effective parameters to extract useful information from the vibration data, which can satisfy the consistency requirement for induction motor fault diagnosis; (2) mutual information technique is an effective tool to reduce the feature space extracted from the CM data, which can achieve a higher recognition rate with selected features; (3) the combination of entropy, mutual information, and support vector machine

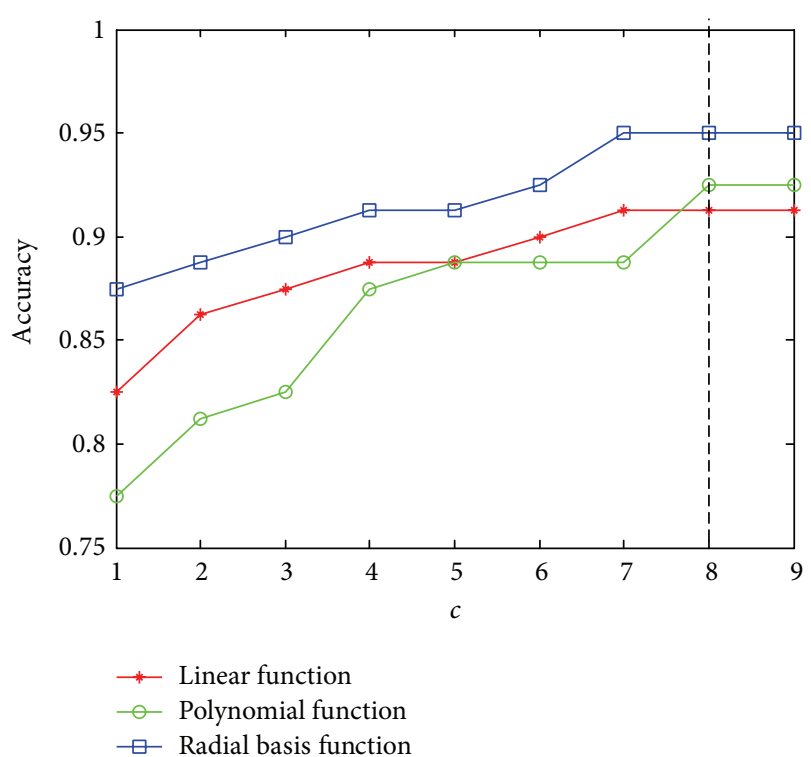

(a) Parameter $c$

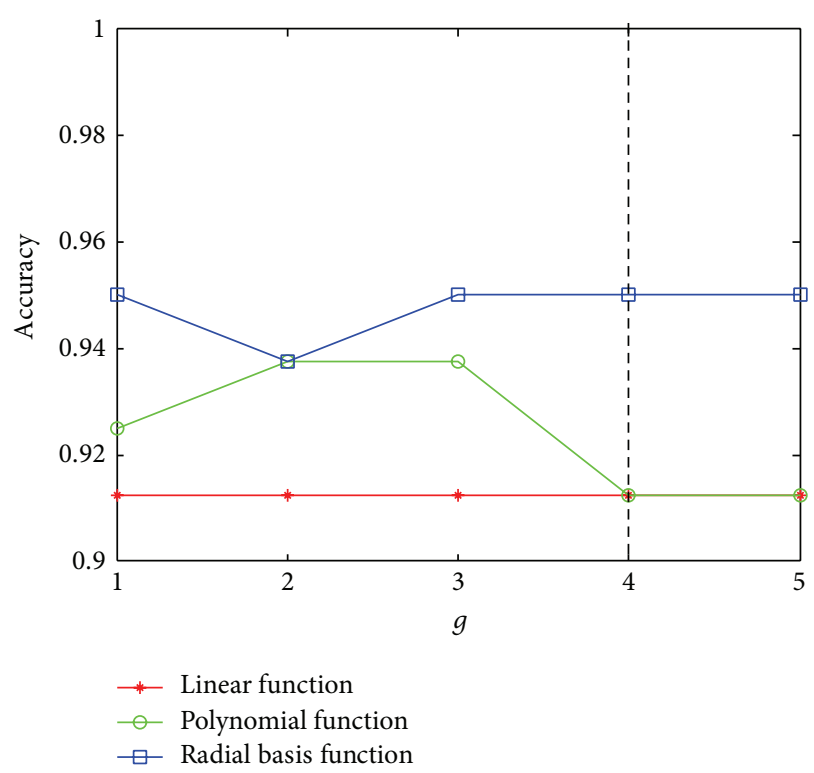

(b) Parameter $g$

FIgURE 11: Classification accuracy of the three SVM models using different parameter values in the data training.

as proposed in the paper can largely improve the effectiveness and accuracy of machine fault classification and diagnosis and thus is very useful in the development of automatic fault diagnosis systems.

\section{Conflict of Interests}

The authors declare that there is no conflict of interests regarding the publication of this paper. 


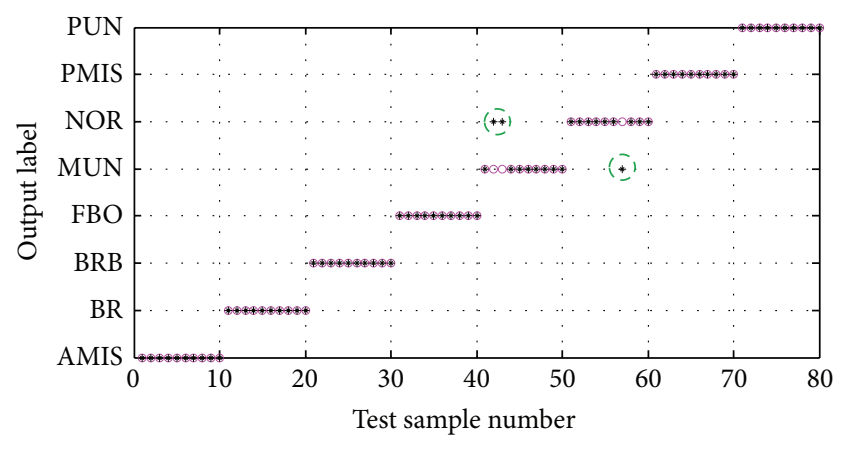

$\circ$ Desired
+ Predicted

FIGURE 12: Accuracy of the proposed model for induction motor fault diagnosis.

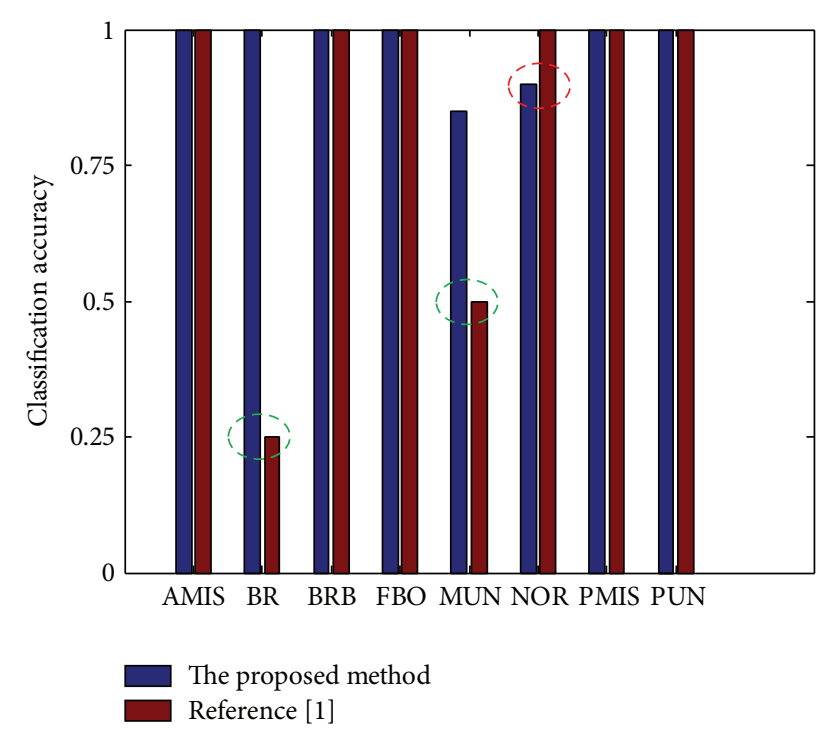

FIGURE 13: Classification accuracy comparison.

\section{Acknowledgment}

This paper is supported by the Fundamental Research Funds for the Central Universities (Grant no. FRF-TP-14-018A2).

\section{References}

[1] T. Han, B.-S. Yang, W.-H. Choi, and J.-S. Kim, "Fault diagnosis system of induction motors based on neural network and genetic algorithm using stator current signals," International Journal of Rotating Machinery, vol. 2006, Article ID 61690, 13 pages, 2006.

[2] T. Han, B.-S. Yang, and Z.-J. Yin, "Feature-based fault diagnosis system of induction motors using vibration signal," Journal of Quality in Maintenance Engineering, vol. 13, no. 2, pp. 163-175, 2007.

[3] Y.-S. Lee, J. K. Nelson, H. A. Scarton, D. Teng, and S. A. Ghannad, "Acoustic diagnostic technique for use with electric machine insulation," IEEE Transactions on Dielectrics and Electrical Insulation, vol. 1, no. 6, pp. 1186-1193, 1994.
[4] W. T. Thomson and M. Fenger, "Current signature analysis to detect induction motor faults," IEEE Industry Applications Magazine, vol. 7, no. 4, pp. 26-34, 2001.

[5] W. T. Thomson, D. Rankin, and D. G. Dorrell, "On-line current monitoring to diagnose airgap eccentricity in large threephase induction motors-industrial case histories verify the predictions," IEEE Transactions on Energy Conversion, vol. 14, no. 4, pp. 1372-1378, 1999.

[6] S. Williamson and K. Mirzoian, "Analysis of cage induction motors with stator winding faults," IEEE Transactions on Power Apparatus and Systems, vol. 104, no. 7, pp. 1838-1842, 1985.

[7] R. R. Schoen, T. G. Habetler, F. Kamran, and R. G. Bartheld, "Motor bearing damage detection using stator current monitoring," IEEE Transactions on Industry Applications, vol. 31, no. 6, pp. 1274-1279, 1995.

[8] F. Thollon, G. Grellet, and A. Jammal, "Asynchronous motor cage fault detection through electromagnetic torque measurement," European Transactions on Electrical Power Engineering, vol. 3, no. 5, pp. 375-378, 1993.

[9] E. R. Filho, R. R. Riehl, and E. Avolio, "Automatic three-phase squirrel-cage induction motor test assembly for motor thermal behavior studies," in Proceedings of the IEEE International Symposium on Industrial Electronics (ISIE '94), pp. 204-209, Santiago, Chile, May 1994.

[10] R. Beguenane and M. E. H. Benbouzid, "Induction motors thermal monitoring by means of rotor resistance identification," IEEE Transactions on Energy Conversion, vol. 14, no. 3, pp. 566570, 1999.

[11] H. Talhaoui, A. Menacer, A. Kessal, and R. Kechida, "Fast Fourier and discrete wavelet transforms applied to sensorless vector control induction motor for rotor bar faults diagnosis," ISA Transactions, vol. 53, no. 5, pp. 1639-1649, 2014.

[12] N. Roveri and A. Carcaterra, "Damage detection in structures under traveling loads by Hilbert-Huang transform," Mechanical Systems and Signal Processing, vol. 28, pp. 128-144, 2012.

[13] N. Roveri, A. Carcaterra, and A. Akay, "Frequency intermittency and energy pumping by linear attachments," Journal of Sound and Vibration, vol. 333, no. 18, pp. 4281-4294, 2014.

[14] Z. Wu and N. E. Huang, "Ensemble empirical mode decomposition: a noise-assisted data analysis method," Advances in Adaptive Data Analysis, vol. 1, no. 1, pp. 1-41, 2009.

[15] Y. Lei, Z. He, and Y. Zi, "Application of the EEMD method to rotor fault diagnosis of rotating machinery," Mechanical Systems and Signal Processing, vol. 23, no. 4, pp. 1327-1338, 2009.

[16] B. Liang, S. D. Iwnicki, and Y. Zhao, "Application of power spectrum, cepstrum, higher order spectrum and neural network analyses for induction motor fault diagnosis," Mechanical Systems and Signal Processing, vol. 39, no. 1-2, pp. 342-360, 2013.

[17] M. Seera, C. P. Lim, D. Ishak, and H. Singh, "Offline and online fault detection and diagnosis of induction motors using a hybrid soft computing model," Applied Soft Computing, vol. 13, no. 12, pp. 4493-4507, 2013.

[18] B. Guo, S. Song, A. Ghalambor, and T. R. Lin, Offshore Pipelines, Gulf Professional Publishing, Oxford, UK, 2nd edition, 2014.

[19] M. Costa, A. L. Goldberger, and C.-K. Peng, "Multiscale entropy analysis of complex physiologic time series," Physical Review Letters, vol. 89, Article ID 068102, pp. 1-4, 2002.

[20] J.-L. Lin, J. Y.-C. Liu, C.-W. Li, L.-F. Tsai, and H.-Y. Chung, "Motor shaft misalignment detection using multiscale entropy with wavelet denoising," Expert Systems with Applications, vol. 37, no. 10, pp. 7200-7204, 2010. 
[21] J. Zheng, J. Cheng, and Y. Yang, "A rolling bearing fault diagnosis approach based on LCD and fuzzy entropy," Mechanism and Machine Theory, vol. 70, pp. 441-453, 2013.

[22] K. Zhu, X. Song, and D. Xue, "A roller bearing fault diagnosis method based on hierarchical entropy and support vector machine with particle swarm optimization algorithm," Measuremen, vol. 47, no. 1, pp. 669-675, 2014.

[23] G. Doquire and M. Verleysen, "Mutual information-based feature selection for multilabel classification," Neurocomputing, vol. 122, pp. 148-155, 2013.

[24] A. Widodo and B.-S. Yang, "Support vector machine in machine condition monitoring and fault diagnosis," Mechanical Systems and Signal Processing, vol. 21, no. 6, pp. 2560-2574, 2007.

[25] S. M. Pincus, "Approximate entropy as a measure of system complexity," Proceedings of the National Academy of Sciences of the United States of America, vol. 88, no. 6, pp. 2297-2301, 1991.

[26] M. Costa, C.-K. Peng, A. L. Goldberger, and J. M. Hausdorff, "Multiscale entropy analysis of human gait dynamics," Physica A: Statistical Mechanics and its Applications, vol. 330, no. 1-2, pp. 53-60, 2003.

[27] J. S. Richman and J. R. Moorman, "Physiological time-series analysis using approximate entropy and sample entropy," American Journal of Physiology-Heart and Circulatory Physiology, vol. 278, no. 6, pp. H2039-H2049, 2000.

[28] M. Costa, A. L. Goldberger, and C.-K. Peng, "Multiscale entropy analysis of biological signals," Physical Review E, vol. 71, no. 2, Article ID 021906, 18 pages, 2005.

[29] D. Hanbay, "An expert system based on least square support vector machines for diagnosis of the valvular heart disease," Expert Systems with Applications, vol. 36, no. 3, pp. 4232-4238, 2009.

[30] M. Saimurugan, K. I. Ramachandran, V. Sugumaran, and N. R. Sakthivel, "Multi component fault diagnosis of rotational mechanical system based on decision tree and support vector machine," Expert Systems with Applications, vol. 38, no. 4, pp. 3819-3826, 2011.

[31] R. Kohavi, "A study of cross-validation and bootstrap for accuracy estimation and model selection," in Proceedings of the International Joint Conference on Artificial Intelligence (IJCAI '95), vol. 2, pp. 1137-1145, Quebec, Canada, August 1995.

[32] G. Niu, T. Han, B.-S. Yang, and A. C. C. Tan, "Multi-agent decision fusion for motor fault diagnosis," Mechanical Systems and Signal Processing, vol. 21, no. 3, pp. 1285-1299, 2007.

[33] G. Niu, A. Widodo, J.-D. Son, B.-S. Yang, D.-H. Hwang, and D.S. Kang, "Decision-level fusion based on wavelet decomposition for induction motor fault diagnosis using transient current signal," Expert Systems with Applications, vol. 35, no. 3, pp. 918928, 2008.

[34] A. Widodo, B.-S. Yang, and T. Han, "Combination of independent component analysis and support vector machines for intelligent faults diagnosis of induction motors," Expert Systems with Applications, vol. 32, no. 2, pp. 299-312, 2007. 


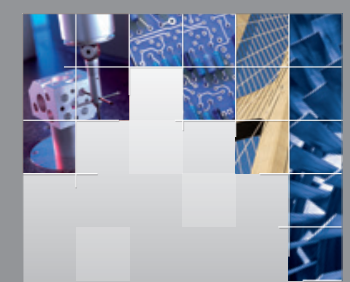

\section{Enfincering}
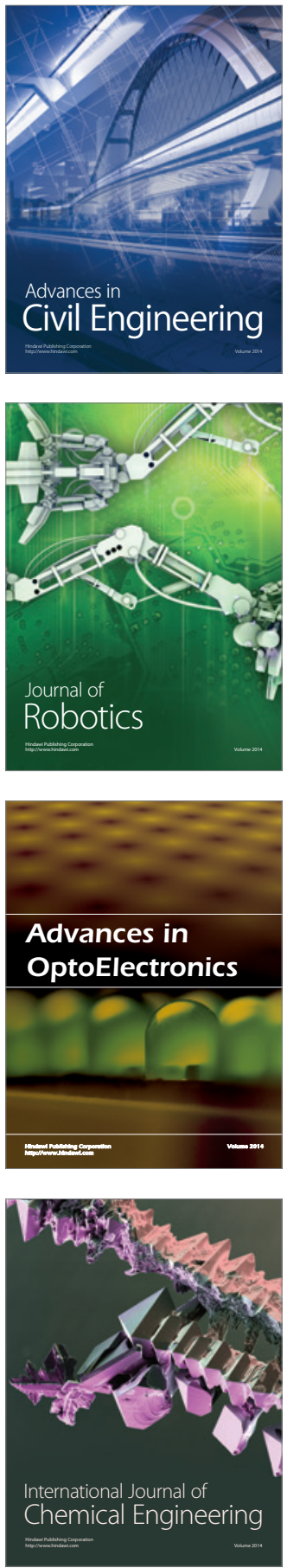

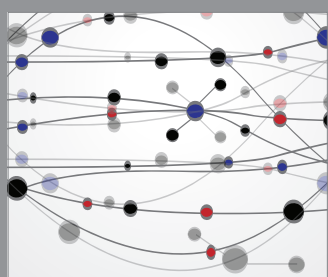

The Scientific World Journal

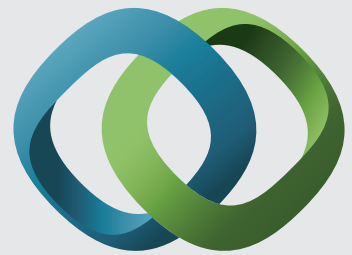

\section{Hindawi}

Submit your manuscripts at

http://www.hindawi.com
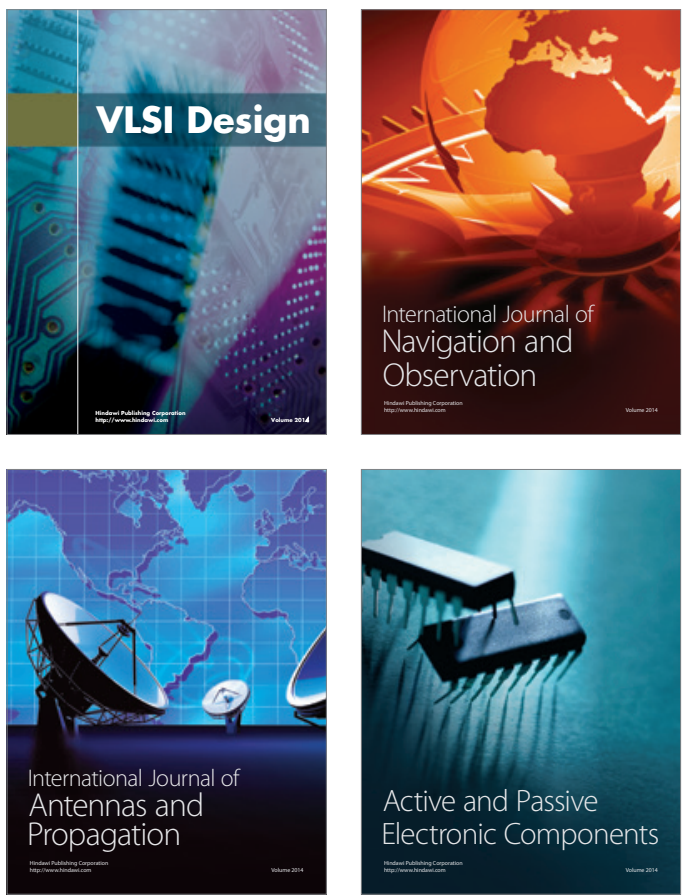
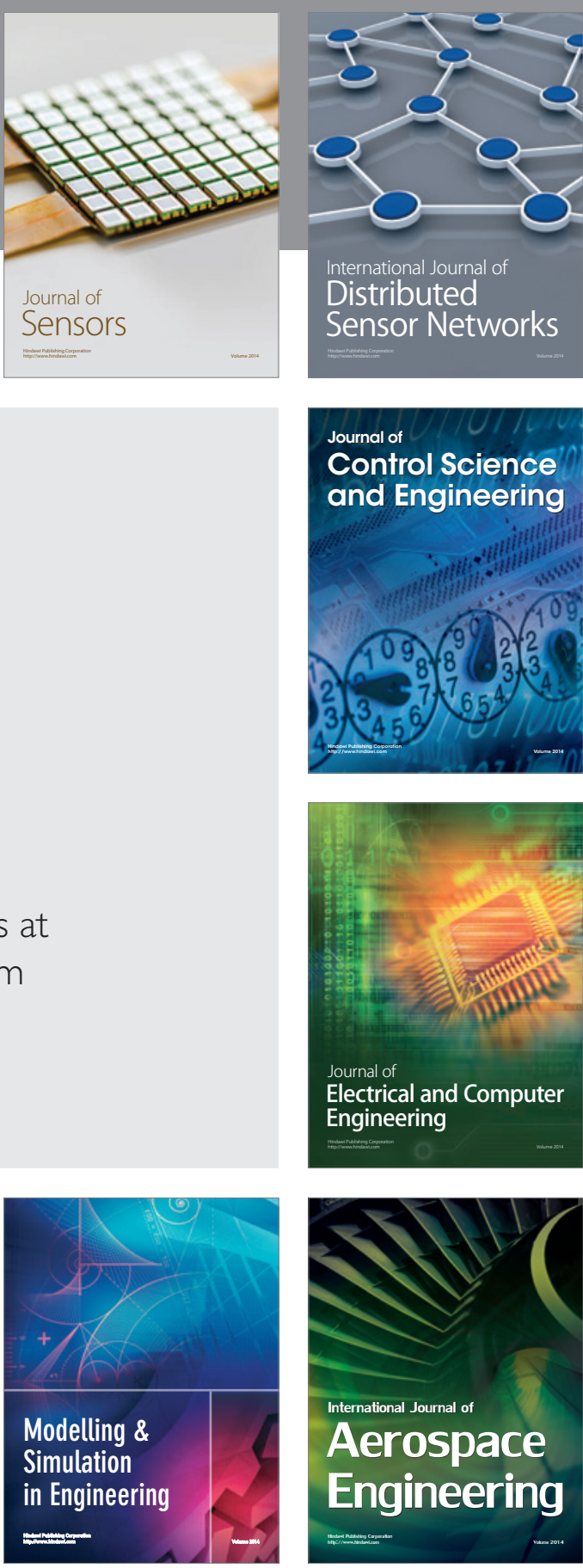

International Journal of

Distributed

Sensor Networks

Journal of

Control Science

and Engineering
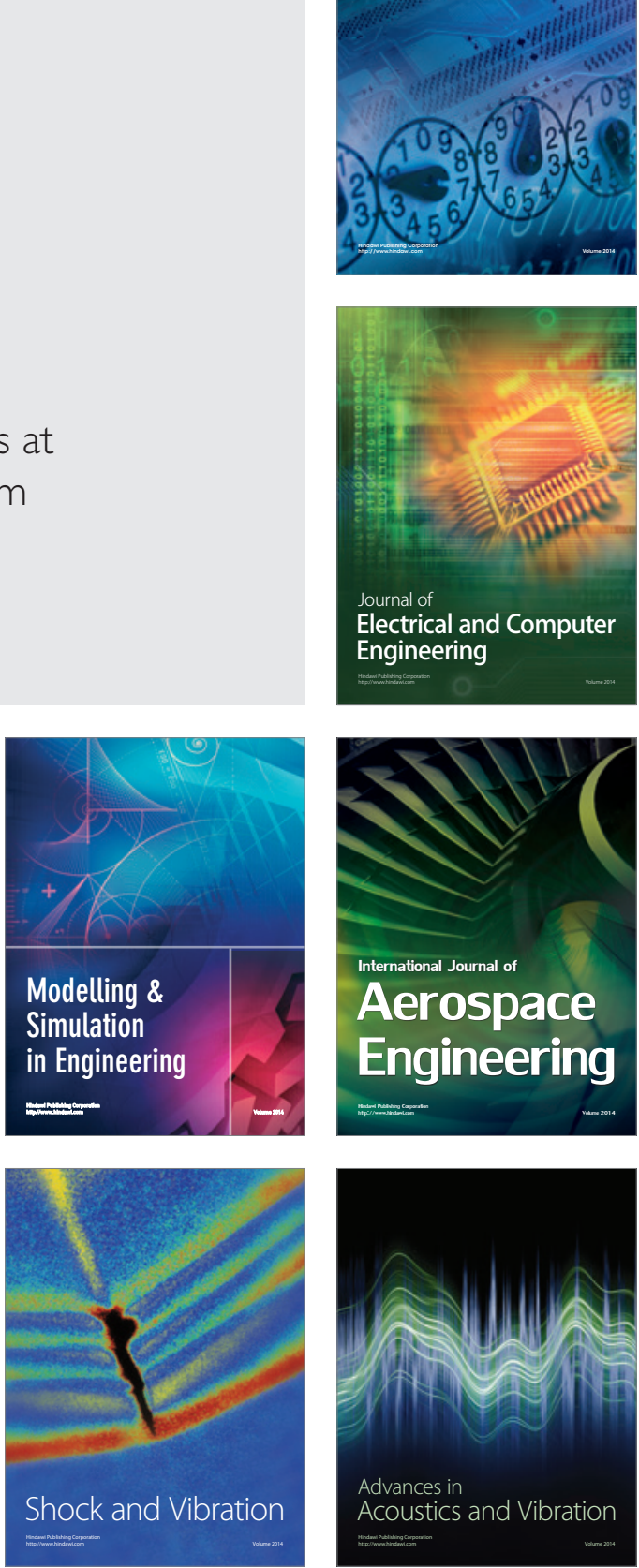